\title{
Effect of transcranial direct current stimulation on sports performance for two profiles of athletes (power and endurance) (COMPETE): a protocol for a randomised controlled trial.
}

Yohan Grandperrin ( $\sim$ yohangp@hotmail.fr )

Centre Hospitalier Universitaire de Besancon https://orcid.org/0000-0001-7697-9989

Sidney Grosprêtre

Universite Bourgogne Franche-Comte

Magali Nicolier

Centre Hospitalier Universitaire de Besancon

Philippe Gimenez

Universite Bourgogne Franche-Comte

Emmanuel Haffen

Centre Hospitalier Universitaire de Besancon

Djamila Bennabi

centre hospitalier universitaire Besancon

Study protocol

Keywords: Transcranial direct current stimulation, Endurance performance, Explosive performance, Dorsolateral prefrontal cortex, Primary motor cortex, Cycling, Parkour

Posted Date: October 17th, 2019

DOl: https://doi.org/10.21203/rs.2.15843/v1

License: (c) (i) This work is licensed under a Creative Commons Attribution 4.0 International License. Read Full License 


\section{Abstract}

Background Transcranial direct current stimulation (tDCS) is promising for improving motor and cognitive performance. Nevertheless, its mechanisms of action are unclear and need to be better characterised according to the stimulated brain area and the type of exercise performed.

Methods/design This is a double-blind cross-over study, organised into two parts: the first is to assess the effects of tDCS on explosive performance (jump task) and the second is to assess the effects on endurance performance (cycling time trial task). Participants, who are recreationally active or athletes (cyclists, parkour practitioners), will receive two active tDCS sessions (over the left dorsolateral prefrontal cortex and right motor cortex) and one sham tDCS session (part A) or two daily tDCS sessions (one active sequence and one sham) over five days (part B). Motor and cognitive performance will be compared before and after the tDCS sessions (part A) and before and after the first session, after the last session and at day 12 and day 30 of each tDCS sequence (part B).

Discussion This study investigates the acute and long-term effects of tDCS on the motor and cognitive performance in healthy subjects. It will try to evaluate if tDCS could be considered as a neuroenhancement technology according to the physical task investigated (endurance versus explosive).

\section{Background}

Over the last decade, neurostimulation techniques have been used for the purpose of improving cognitive and psychomotor functions in healthy subjects. Among them, transcranial direct current stimulation (tDCS) is a safe, low-cost, portable, non-invasive neuromodulation technique that delivers low-intensity, direct current to cortical areas. tDCS induces changes in cortical excitability that can last from a few minutes to several hours after the stimulation $[1,2]$.

In addition, tDCS could also improve exercise performance and reduce neuromuscular fatigue. A recent meta-analysis by Machado et al. assessed the effects of tDCS on performance improvement during different exercises (muscle strength exercise or whole body dynamic cyclic exercise). Eleven studies ( $\mathrm{N}=236$ participants) were included in this quantitative analysis. The authors found weak evidence of a beneficial effect of anodal tDCS applied over the primary motor cortex [M1] before the cycling time to exhaustion, while cathodal stimulation had no detrimental effect on cycling performance. It should be noticed that these results are strongly influenced by a single study. Moreover, anodal tDCS would not have any effect on the isometric strength of the upper or lower limbs and few studies have evaluated the effects of tDCS on isokinetic or dynamic muscle strength [3]. To sum up, many studies have demonstrated a positive enhancement of performance using tDCS [4-6] while others failed to find any improvement [7-9] ; therefore, no particular consensus could be made based on the literature. Several factors may explain such a 
disparity in these results, from the type of physical or cognitive task used to the montage of the electrode.

First, tDCS was used to enhance different types of exercise (e.g., isometric, dynamic, or isokinetic strength, cycling) where the intensity could vary according to the protocol (e.g., cycling time to exhaustion, trial cycling time). According to Angius et al. [10], it is necessary to separate studies with single-joint exercise and whole-body exercise due to differences in the cardiorespiratory, metabolic and neuromuscular responses [11]. It also seems appropriate to separate endurance and explosive tasks. Basically, explosive efforts require the production of maximum power output over a minimum amount of time, while endurance tasks require effort management to maintain a targeted submaximal level of performance over a long period of time. tDCS could, thus, improve motor performance by different ways depending on the type of exercise practised; increase in the maximal power output, decrease in the perceived exertion, or modulation of pacing strategies.

Second, the discrepancy of the stimulation's parameters (electrode placement, current intensity, and density, the timing of tDCS (online versus offline)) from one study to another could explain the conflicting results obtained in previous literature. Accordingly, tDCS configuration seems to be a major factor to explore. Angius et al. have compared cephalic (the anode over the left M1 and the cathode over the right dorsolateral prefrontal cortex [dlPFC]) and extracephalic (the anode over the left M1 and the cathode over the shoulder) tDCS montage and have found an improvement of the isometric endurance performance of the lower limb with the extracephalic montage. Changes in the current direction and negative effects of the return electrode would explain the differences between the two montages [5].

The mechanisms of action of tDCS, therefore, remain unclear. Since they could widely depend on the stimulated brain region, two areas attract our attention-M1 and dlPFC. Regarding the endurance tasks, neuromuscular fatigue is multifaceted and is influenced by both central and peripheral factors. Peripheral fatigue results in changes at or distal to the neuromuscular junction whereas central fatigue represents the inability of the central nervous system [CNS] to generate or maintain central activation of the muscle. Central fatigue is accompanied by changes in the activity of the spinal motoneurons (spinal fatigue) and a reduction in the motor cortical neuronal drive (supraspinal fatigue) $[12,13]$. If tDCS over the M1 modulates the corticospinal output [14], it could increase the excitability of the motor areas and delay the detrimental effects of fatigue over the neural drive of active muscles. In regards to those considerations, the prefrontal cortex [PFC] could also be a target to improve performance. In fact, PFC increases neuronal activation to reinforce muscle force during an exercise [15]. It may have a motivational function and a role in pacing strategies $[16,17]$. tDCS over the PFC could improve motivation and inhibit or reduce negative external factors (e.g., muscle pain), 
leading to a gain in performance. More particularly, due to the key role of the left dlPFC in cognitive control, decision-making and approach motivation, and the benefit of tDCS effects on cognitive function, this latter appears the main area of interest [18-21]. Since the excitability of both of these areas could theoretically be modulated to increase maximal power output, the question of the response to tDCS in terms of explosive performance remains open. In fact, despite rare clues in the literature showing a positive effect on vertical jump performance [22], no studies really assessed the tDCS effects over such type of exercise and its associated mechanisms. Indeed, regarding the tDCS effects on physical tasks, the literature is more developed regarding endurance performance.

To clarify the tDCS effect on these different types of neuromuscular performance, we propose to evaluate the acute and long-term effects of tDCS during two tasks: an explosive task (jumps) and an endurance task (20 minutes cycling trial). The main objective is to compare the effects of tDCS applied over the left dlPFC with sham tDCS on the performance of the neuromuscular system. In the present protocol, the choice has been made to recruit participants with different backgrounds regarding sports practice, from sedentary people to high-level athletes in the targeted performance (cyclists for endurance or parkour athletes for explosive performance). Indeed, the effect of tDCS on physical performance could also be functional of the initial level [23].

This study is comprised of a monocentre sham-controlled randomised crossover double-blinded cross-over trial, comparing active tDCS versus sham tDCS. It is divided into two parts: part A with the jumpers and part B with the cyclists. We hypothesise that the active tDCS sessions would improve motor performance and reduce neuromuscular fatigue. We will seek a better understanding of the factors that could vary the effects of tDCS: stimulated brain area, type of exercise (enduring vs. explosive), tDCS configuration (cephalic vs. extracephalic montage), and the number of tDCS sessions (single vs. repeated stimulation).

\section{Method/design}

\section{Study setting and overview}

This research will be carried out in collaboration between the psychiatric department of Besançon university hospital and the scientists of the Sports Faculty of the University of Besançon (C3S Laboratory). All experiments will take place on the EPSI research platform (Entraînement Performance Santé Innovation, Besançon). This protocol is divided into two parts to explore the effects of tDCS by type of exercise:

- Part A which assesses the tDCS effects on explosive performance (jumps).

- Part B which assesses the tDCS effects on endurance performance (cycling time trial). 
Fifty subjects (20 in part A, 30 in part B) will be recruited from the sports university or sports federated organisations. For part A, athletes practicing parkour, an activity that consists of jumping obstacles in various environments, have been chosen as they usually present a very explosive neuromuscular profile [24]. For part B, amateur and professional cyclists will be recruited.

After information about the study, written informed consent will be obtained.

In part A, subjects will be divided into two groups (amateur versus high-level practice) and receive three sessions of tDCS (active over the left dlPFC, active over the right M1 and sham over the left dlPFC). The order of the sessions will be randomised (Figure 1).

Figure 1: Study flow diagram (part A)

In part B, subjects will be divided into three groups (sedentary, amateur, and high-level practice). They will receive two sequences of tDCS divided in two daily active tDCS sessions (over dlPFC) over five days and after a wash-out of one month, two daily sham tDCS sessions over five days or vice and versa (Figure 2).

Figure 2: Study flow diagram (Part B)

Common measurements to part A and B include a clinical assessment of impulsivity based on self-report scales and behavioural tasks, task-based measures of motivation, and an assessment of neuromuscular function (Electromyographic recordings and evoked potentials from nerve percutaneous stimulation). These measures will be performed before and after each brain stimulation session (part A) or before and after each brain stimulation sequence, and at day 12 and day 30 (part B).

For each group, baseline measures will include a clinical assessment of depression-severity based on self-reports and clinical rated scales. These data will be compared to those obtained after the last tDCS session and at day 12 and day 30 (part B). 
In order to assess the tDCS effects on two types of physical performances, the physical tasks differ from part A to part B. In part A, the performances assessed are jumping tasks (squat jump [SJ], countermovement jump [CMJ], and standing long jump [SLJ]), while in part $\mathrm{B}$, the endurance task (20 minutes cycling time trial) is performed. The endurance task is performed maximally pre and post-tDCS sequence, then at day 12 and day 30, while it is performed sub-maximally (60\% of peak power) during each tDCS training session (i.e., twice a day for five days). In part B, other measures will be performed at the start, immediately after the last session of tDCS and then at day 12 and day 30 (see Study Procedure for details). After unblinding, active and sham stimulation outcomes will also be compared.

\section{Inclusion criteria}

Eligible subjects will be invited to take part in this trial according to the following criteria: (1) male and female subjects over 18 years old; (2) right-handed; (3) absence of addictive comorbidities (except tea, coffee, tobacco) and severe progressive neurologic and somatic and psychiatric disease; (4) Part A: Amateur jump practice (less than 4000 hours of practice during the last five years) or high-level jump practice (more than 4000 hours of practice during the last five years); OR

(5) Part B: High-level cycling practice (more than 4000 hours of practice during the last five years), amateur cycling practice (less than 4000 hours of practice during the last five years), or sedentary (less than two hours of recreational sports practice by week).

\section{Exclusion criteria}

Subjects will be excluded if they are identified as having any of the following: (1) younger than 18 years of age; (2) left-handed; (3) presence of psychiatric or addictive diseases; (4) presence of severe somatic or progressive neurologic pathologies; (5) low cooperation stated by the investigator; (6) pregnancy; (7) concurrent participation in another trial; (8) no coverage by the national health insurance; and (9) measure of protection or guardianship of justice.

\section{Interventions}

Transcranial Direct Current Stimulation: 
Direct current will be delivered by a neurostimulator system (StarStim ${ }^{\circledR}$, Neuroelectrics $@$, Barcelona, Spain) that allows a sham and double-blind mode. It will be transmitted by two saline-soaked synthetic sponge electrodes (Sponstim ${ }^{\circledR}, 25 \mathrm{~cm}^{2}$ ), placed in a neoprene head cap.

In part $\mathrm{A}$, subjects will benefit from three 20-minute sessions of tDCS (two active and one sham), separated by a minimum of 48 hours. Electrode placement will be realised according to the EEG 10-20 International System (see Table 1) and sequence order will be determined by computer randomisation.

\begin{tabular}{l|c|c|c|}
\hline PART A & \multicolumn{3}{|c|}{ Sequence order determined by randomisation } \\
\hline Anode & F3 over left dlPFC & $\begin{array}{c}\mathrm{FC}_{2} \text { over right } \\
\text { motor cortex }\end{array}$ & $\begin{array}{c}\text { Sham : F3 over left } \\
\text { dlPFC }\end{array}$ \\
\hline Cathode & $\begin{array}{c}\text { AF8 over right } \\
\text { supraorbital region }\end{array}$ & $\begin{array}{c}\text { Controlateral } \\
\text { shoulder }\end{array}$ & $\begin{array}{c}\text { AF8 over right } \\
\text { supraorbital region }\end{array}$ \\
\hline $\begin{array}{l}\text { Intensity of } \\
\text { stimulation }\end{array}$ & 2 milliAmpers & 2 milliAmpers & 0 milliAmper \\
\hline
\end{tabular}

Table 1: Electrode placement and stimulation parameters in Part A.

In part $B$, subjects will benefit from two sequences of 20-minutes tDCS sessions per day, for five days consecutively (either, a sequence of 10 active or 10 sham sessions according to the computer randomisation). After a wash-out of one month, they will receive the second sequence (cross-over). The anodal electrode will be placed over F3 (left dlPFC) and cathodal electrode over AF8 (right supraorbital region). The intensity of stimulation is 2 $\mathrm{mA}$ (in active sequence) or $0 \mathrm{~mA}$ (sham sequence).

For sham stimulation, in each part of the protocol, the current will gradually ramp up over 30 seconds until $2 \mathrm{~mA}$ at the beginning of the stimulation and will then gradually ramp down over an equal amount of time at the end, thus leading to the same initial and final sensations of active tDCS.

\section{Neuromuscular assessment:}

The general performance of the neuromuscular system will be assessed on plantar flexors. Therefore, maximal force production capacity will be assessed through both the plateau of isometric maximal voluntary contractions and the rate of force development, i.e., the slope of the rising force-time curve.

Electromyographic (EMG) activity of plantar-flexor muscles will be recorded continuously during motor tasks (maximal voluntary contractions, jumps and cycling time trial). EMG activity is recorded from four muscles of the right leg (soleus, SOL; medial gastrocnemius, MG; tibialis anterior, TA; vastus lateralis: VL). Before electrode placement, the skin is first shaved and dry-cleaned with alcohol to keep low impedance $(<5 \mathrm{k} \Omega)$. The EMG signal is recorded with Trigno sensors (Delsys, Natick, Massachusetts, USA). 
The nerve-evoked potentials are elicited to account for the relative contributions of the several nervous levels to the possible changes induced by tDCS. The evolution of these evoked potentials following acute or chronic interventions is commonly assessed to account for neuromuscular changes, particularly at spinal level [25].

The posterior tibial nerve is stimulated through single rectangular pulses (1-ms width) delivered by Digitimer stimulators (model DS7A, Hertfordshire, UK). Stimulations are elicited with a self-adhesive cathode (8-mm diameter, Ag-AgCL) placed in the popliteal fossa, and an anode (5 x $10 \mathrm{~cm}$, Medicompex SA, Ecublens, Switzerland) placed over the patella.

The intensity of the stimulation is then progressively increased from the SOL, MG, and LG H-reflex threshold to find different targeted intensities. Three different responses are recorded and taken for analysis: the H-reflex, the maximal muscle compound action potential (M-wave) and the V-wave. The H-reflex is a classical tool to investigate the spinal excitability by reflecting the efficiency of the Ia-to-alpha motoneuronal transmission. The Vwave characterizes the magnitude of the neural drive from M1 addressed to the spinal motoneuronal pool. The aim of recording the maximal M-wave is twofold: it serves as a marker of the excitability of the neuromuscular junction, and is used to normalise each of the other responses.

In part $A$, the neuromuscular assessment will be performed before and after each tDCS session. In part B, it will be performed before and after the first sequence of tDCS, then at day 12 and day 30 for each sequence of the cross-over.

\section{Explosive or endurance task:}

In part A, three types of jumps (SJ, CMJ, and SLJ) will be performed by the subjects, before and after each tDCS session. After a warm-up, subjects will benefit from two trials per jump. The order of the jumps will be randomised.

Height and suspension time of vertical jumps will be assessed by means of a force-plate (Kistler Instruments, AG). Horizontal jumps are measured with a standing long jump mat.

For vertical jumps, participants are asked to jump and land with both feet simultaneously on the force platform, with no initial steps or shuffling. Angles of the knee and ankle are visually controlled during all landings. The SJ was assessed from a starting position with knees flexed at $90^{\circ}$ and weight well distributed over both feet. Participants are asked to keep their trunk straight, and no counter movement with the legs is allowed. For the CMJ performances, participants begin in an upright standing position. They are asked to bend to $90^{\circ}$ knee flexion and immediately jump without pausing in the squat position.

In part $\mathrm{B}$, the endurance task will be performed maximally pre and post-tDCS sequence, then at day 12 and day 30 of each sequence while it will be performed sub-maximally (at $60 \%$ of maximal power) during each tDCS training session (i.e., twice a day for five days). 
Maximal power of each subject will be determined during the first cycling time trial (Day 1).

During this effort, no feedback is displayed. Subjects will not have access to the effortrelated parameters (speed, distance covered, etc) except for the time remaining. During the performance, the different measures are recorded (myoelectrical activity, power output, heart rate, pedalling rate, Cook and Borg CR 10 scales).

A habituation session will be conducted at the time of inclusion.

\section{Outcomes}

Our main efficacy criteria will be the evaluation of neuromuscular performance during an explosive task (part A) or during an endurance task (part B).

In part $A$, we will compare the height of the vertical jumps or the length of horizontal jumps (in centimetres) performed before and after active or sham tDCS session. In part B, endurance performance will be assessed by the comparison of the average power output during a time-trial realised before, after the first, on the last tDCS session, and at day 12 and day 30 of each sequence.

Secondary efficacy criteria will comprehend:

1. tDCS effects on the neuromuscular system by analysis of the EMG signals, spinal excitability, and voluntary activation, electromechanical efficiency and comparison of the results obtained before and after tDCS session (jumpers) or before and after the first and the last tDCS session, and at day 12 and day 30 of each sequence (cyclists).

2. tDCS effects according to the motor expertise by comparison of performance between subjects with an amateur or high-level jump practice, or sedentary subjects and those who have an amateur or high-level cycling practice.

3. tDCS effects on motor gestures and speed-accuracy trade-off during a pointing task before and after the tDCS session (jumpers).

4. Changes in the Rating of Perceived exertion and muscle pain, by comparison of the scores from the Borg CR10 scale and the Cook's scale obtained during the endurance task (every five minutes) before and after the first and the last tDCS session and at day 12 and day 30 of each sequence (cyclists).

5. Changes in motivation, by comparison of scores from the Effort Expenditure for Rewards Task [EEfRT] obtained before and after the tDCS session (jumpers) or before and after the first and the last tDCS session and at day 12 and day 30 of each sequence (cyclists).

6. tDCS effects on impulsivity by comparison of the scores from the French version of the Barratt Impulsiveness Scale [BIS-10], the experimental Go/No-Go and Stroop tasks and the Balloon Analog Risk Task [BART] obtained before and after the tDCS session 
(jumpers) or before and after the first and the last tDCS session, and at day 12 and day 30 of each sequence (cyclists).

7. Changes in delay discounting, by comparison of the scores from the French version of the Monetary Choice Questionnaire (MCQ) obtained before and after the tDCS session (jumpers) or before and after the first and the last tDCS session and at day 12 and day 30 of each sequence (cyclists).

\section{Study procedure:}

In part A, the study will have four phases. During the first phase, subjects will be recruited by the sports research team. Information about the study, the neurostimulation technique and the objectives of the research will be given to each subject by a trained psychiatric investigator. Enrolment and timetable of visits are scheduled with the volunteers.

The detailed procedure is displayed in Figure 3.

Other phases will correspond to both inclusions of volunteers and visits for the experimental session. After the informed consent is signed, a clinical exam is conducted in order to verify the inclusion and exclusion criteria. Following the inclusion, subjects are randomly assigned using a computer-generated list in order to determine the sequence of stimulation (over M1, over dlPFC and sham). Predefined codes assigned to either the active or sham stimulation will be used by the psychiatry staff to start the stimulator, allowing a double-blind study design. The three visits will be organised in the same way and separated by at least 48 hours. Cognitive, motor tasks and neuromuscular assessments will be realised before and immediately after the stimulation.

Figure 3: Randomised cross-over design for COMPETE (part A) (Standard Protocol Items: Recommendations for Interventional Trials (SPIRIT) Figure). 


\begin{tabular}{|c|c|c|c|c|c|}
\hline \multirow[b]{4}{*}{ TIMEPOINT (DAYS) } & \multicolumn{5}{|c|}{ STUDY PROTOCOL } \\
\hline & \multirow{2}{*}{$\begin{array}{c}\text { Phase } 1 \\
\text { Enrolment }\end{array}$} & \multicolumn{2}{|c|}{ Phase 2} & $\begin{array}{c}\text { Phase } \\
3\end{array}$ & $\begin{array}{c}\text { Phase } \\
4\end{array}$ \\
\hline & & \multirow{2}{*}{$\frac{\text { Inclusion }}{\text { Day } 0}$} & \multicolumn{3}{|c|}{ Follow-up } \\
\hline & Day -1 & & $\begin{array}{c}\text { Visit } \\
1 \\
\end{array}$ & $\begin{array}{c}\text { Visit } \\
2 \\
\end{array}$ & $\begin{array}{c}\text { Visit } \\
3 \\
\end{array}$ \\
\hline$\overline{\text { ENROLMENT }}$ & \multirow{4}{*}{$\mathrm{X}$} & \multirow{4}{*}{$\begin{array}{l}\mathrm{X} \\
\mathrm{X}\end{array}$} & & & \\
\hline Presentation of study & & & & & \\
\hline Informed consent & & & & & \\
\hline Randomised allocation & & & & & \\
\hline $\begin{array}{l}\text { INTERVENTIONS } \\
\text { Active tDCS } \\
\left(2 \mathrm{~mA} / 25 \mathrm{~cm}^{2}, 20 \mathrm{~min}, \mathrm{dlPFC}\right)\end{array}$ & & & \multirow{3}{*}{\multicolumn{3}{|c|}{$\begin{array}{l}\text { SEQUENCE } \\
\text { DETERMINED BY } \\
\text { RANDOMISATION }\end{array}$}} \\
\hline $\begin{array}{l}\text { Active tDCS } \\
\left(2 \mathrm{~mA} / 25 \mathrm{~cm}^{2}, 20 \mathrm{~min}, \mathrm{M} 1\right)\end{array}$ & & & & & \\
\hline Sham tDCS & & & & & \\
\hline ASSESSMENTS & & \multirow{4}{*}{$\mathrm{X}$} & \multirow{4}{*}{$\mathrm{X}$} & \multirow[b]{3}{*}{$\mathrm{X}$} & \multirow[b]{3}{*}{$\mathrm{X}$} \\
\hline Clinical evaluation & & & & & \\
\hline Primary outcome : Height or length of jumps & & & & & \\
\hline $\begin{array}{l}\text { Secondary outcome: Neuromuscular parameters, } \\
\text { BIS-10, BART, MCQ, EEfRT, Go/No-Go task, Stroop task, } \\
\text { QIDS-C16, QIDS-SR16, Pointing task }\end{array}$ & & & & $\mathrm{x}$ & $\mathrm{X}$ \\
\hline
\end{tabular}

BART Balloon Analog Risk Task, BIS-10 Barratt Impulsiveness Scale-10, dlPFCDorsolateral Prefrontal Cortex, EEfRTEffort Expenditure for Rewards Task , M1 Primary motor cortex, MCQ Monetary Choice Questionnaire, QIDS-C16 16-Item Quick Inventory of Depressive Symptomatology, Clinician Rating , QIDS-SR16 16-Item Quick Inventory of Depressive Symptomatology, Self-Report

In part $B$, the study is comprised of three phases (the detailed procedure displayed in Figure 4). The first phase corresponds to the recruitment, conducted in the same way as in Part A. The second phase relates to both the inclusion of the volunteers and the period of the first tDCS sequence. Behavioural scores and neuromuscular parameters will be collected at baseline and immediately at the first tDCS session. It will be delivered on a Monday and two daily sessions will be performed during the following days (during the endurance task) up to Friday. Clinical, neuromuscular and behavioural assessments will be realized once the last tDCS session has been delivered (Day 5), and then at day 12 and day 30. 
After the last assessment, cross-over will be realised: subjects who underwent sham stimulation sessions will be then submitted to active sessions and vice-versa (third phase) with the same design.

Figure 4. Randomized cross-over design for COMPETE (part B) (Standard Protocol Items: Recommendations for Interventional Trials (SPIRIT) Figure).

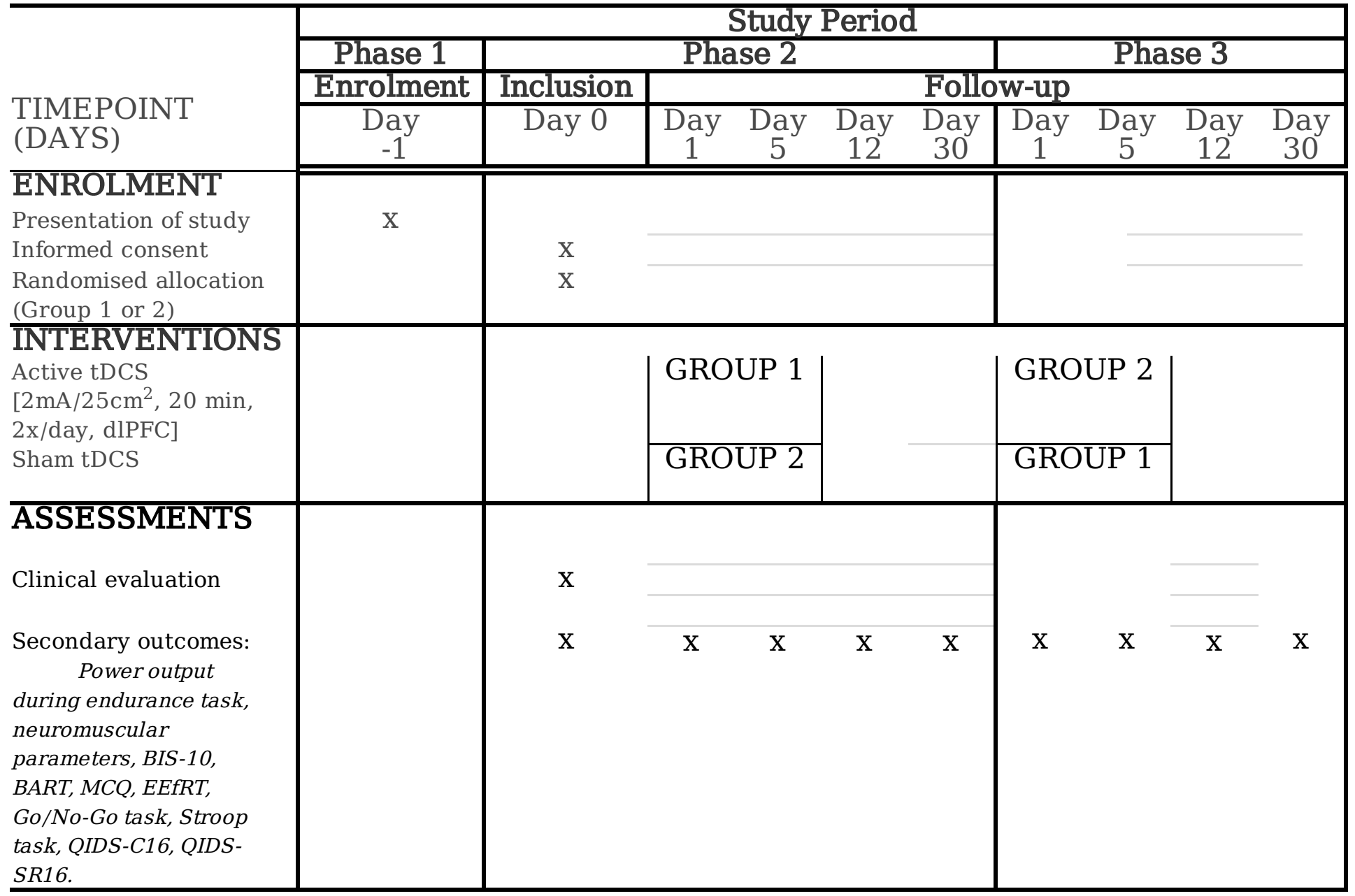

BART Balloon Analog Risk Task, BIS-10 Barratt Impulsiveness Scale-10, dlPFC Dorsolateral Prefrontal Cortex, EEfRTEffort Expenditure for Rewards Task, M1 Primary motor cortex, MCQ Monetary Choice Questionnaire, QIDS-C16 16-Item Quick Inventory of Depressive Symptomatology, Clinician Rating, QIDS-SR1616-Item Quick Inventory of Depressive Symptomatology, Self-Report

\section{Sample size}

Our sample size calculation is based on the primary efficacy outcome that relates to changes in motor performance before and after a tDCS session during an explosive task (jumps). In a previous study, Lattari et al. [22] showed an $11.2 \%$ improvement in the performance of the CMJ following a tDCS session, with a standard deviation for paireddifferences of 6.5 . We expect a 15\% improvement in our study due to an additional experimental session. Considering an autocorrelation of $50 \%$ between measures in the 
same subject, a significance level of $5 \%$ and a power of $90 \%, 20$ jumpers are included to meet the objectives of the study.

\section{Withdrawal of Consent}

Participants will be informed that taking part is completely voluntary and that they are free to withdraw from the study at any time without prejudice and without having to give a reason. They may also be removed at any time from the study if adverse events or any exclusion criteria will be detected. If a disease is discovered during the study, subjects will be proposed with a medical follow-up adapted to it.

\section{Data management and statistical analyses}

All collected information will be registered in physical files (CRFs: Case Report Files), previously anonymised with the participant's randomization code in order to respect confidentiality at all times. Computer test data (BART, EEfRT) and physical test results will be collected in electronic format.

All researchers and trained staff called upon to collaborate in the tests are bound to secrecy.

All randomised subjects will be used for the efficacy analyses. Qualitative variables will be described in terms of effective, absolute and relative frequencies for each modality. Quantitative variables will be described in terms of minimum and maximum, quartiles, means and standard variations.

In part $\mathrm{A}$, to compare the performance between sham and active stimulation, a paired Student's t-test or the Wilcoxon test according to the normality of the distribution (ShapiroWilk test) is used.

Statistical comparisons between groups (active tDCS or sham) is used for the secondary efficacy criteria:

- At each time the Student test for Gaussian quantitative variables in the case where normality is verified (Shapiro-Wilk test) and the repeated measures ANOVA taking into account the different study times.

- The Wilcoxon non-parametric test or the Kruskall-Wallis test for semi-quantitative or quantitative non-Gaussian variables.

- The McNemar's test for qualitative variables.

In part $B$, the repeated measures ANOVA to compare different results between active and sham stimulation is used.

Analyses will be performed using the SAS ${ }^{\circledR} 9.4$ Software for Windows (SAS Institute $\odot$, Cary, North Carolina, USA). 


\section{Monitoring:}

Data monitoring will be realised by a Clinical Research Associate designed by the University Hospital of Besançon. He will have documented competence to follow up the research and no competing interests. He will annually verify adequate progress of the research and compliance with ethical rules.

Any adverse events occurring after the consent signing will be reported to the requesting authority. The ENNOV software will be used for the declaration and the University Hospital of Besançon will be in charge of informing national health surveillance agencies.

\section{Ethics and dissemination}

The study is prospectively registered on ClinicalTrials.org as "Effect of tDCS on Sport Performance for Two Categories of Athletes: Explosive Profile and Enduring Profile", identifier NCT03937115 (available on: https://clinicaltrials.gov/ct2/show/NCT03937115). This protocol is approved by the French Committee for the Protection of Persons Est IV, under the number 18/47. It adheres to the Standard Protocol Items: Recommendations for Interventional Trials (SPIRIT) guidelines (see Additional File 1: SPIRIT Checklist COMPETE).

Prior to enrolment, the principal investigator will provide full information about the study to the volunteers. If they agree to participate, they will sign a written informed consent. Subjects will be informed that taking part is completely voluntary and that they are free to withdraw from the study at any time without prejudice and without having to give a reason.

Data management and monitoring respect the French Jardé Law (No. 2012-300, from 5 March 2012) and the French Public Health Code's guidance on good clinical practice to conduct trials of human participants.

Dissemination will be provided by the research team through presentations at conferences and scientific publications.

\section{Discussion}

COMPETE is an ambitious protocol that will seek to understand the acute and long-term effects of tDCS on physical performance. It will compare the tDCS effects on two types of exercises (explosive vs. endurance) using a different administration mode (single vs. repeated sessions). 
The challenge of this study will be to specify the action mechanisms of tDCS on performance according to the exercise type, the stimulated brain area, the tDCS configuration type and the level of athletes included.

To the best of our knowledge, this study will be the first to assess the effect of tDCS on different sport performances by gathering psychometric and neuromuscular measurements. It will determine whether and how the improvement or disruption of the cognitive dimensions studied (mood, motivation and impulsivity) could affect explosive and/or endurance performance.

Finally, some authors have already argued that tDCS may be considered as a new form of doping. Many athletes use tDCS during training for several hours [26] although the effects of prolonged administration are unclear. Our study could determine if chronic use involves risks for them and raise the question of legislation around the free use of tDCS.

\section{Trial Status}

The study is recruiting subjects from November 2018 until April 2020, aiming to enroll 50 subjects. This protocol is version 2.0, 29 May 2018.

\section{Abbreviations}

$\begin{array}{ll}\text { BART } & \text { Balloon Analog Risk Task } \\ \text { BIS-10 } & \text { Barratt Impulsiveness Scale-10 } \\ \text { CMJ } & \text { Countermovement Jump } \\ \text { CNS } & \text { Central Nervous System } \\ \text { dlPFC } & \text { Dorsolateral Prefrontal Cortex } \\ \text { EEfRT: } & \text { Effort Expenditure for Rewards Task } \\ \text { EEG } & \text { Electroencephalography } \\ \text { EMG } & \text { Electromyography } \\ \text { M1 } & \text { Primary motor cortex } \\ \text { MCQ } & \text { Monetary Choice Questionnaire } \\ \text { MG } & \text { Medial Gastrocnemius } \\ \text { PFC } & \text { Prefrontal Cortex } \\ \text { QIDS-C16 } & \text { 16-Item Quick Inventory of Depressive Symptomatology, Clinician Rating } \\ \text { QIDS-SR16 } & \text { 16-Item Quick Inventory of Depressive Symptomatology, Self-Report } \\ \text { SJ } & \text { Squat Jump } \\ \text { SLJ } & \text { Standing Long Jump } \\ \text { SOL } & \text { Soleus } \\ \text { TA } & \text { Tibialis Anterior }\end{array}$


tDCS Transcranial Direct Current Stimulation

VL Vastus Lateralis

\section{Declarations}

\section{Ethics approval and consent to participate}

This protocol is version 2.0, 29 May 2018 and adheres to the Standard Protocol Items:

Recommendations for Interventional Trials (SPIRIT) guidelines.

Ethical approval has been confirmed from the French Committee for the Protection of Persons Est IV (number 18/41).

Prior to enrolment, the principal investigator will provide full information about the study to the volunteers. If they agree to participate, they will sign a written informed consent.

\section{Consent for publication}

Not applicable

\section{Availability of data and materials}

Data sharing is not applicable to this article as datasets were not yet generated during the current study.

\section{Competing interests:}

The authors declare that they have no competing interests.

\section{Funding}

This study was supported by a grant from the University Hospital of Besançon and Burgundy-Franche-Comte region (Call of Project APICHU RBFC 2018) in France (protocol ID 2018-A00755-50).

\section{Authors' contributions}

YG and SG are co-first authors, they contributed equally to this work. YG, SG and DB contributed to manuscript preparation and revision. MN, PG and EH contributed to critical revision. All authors read and approved the final manuscript.

\section{Acknowledgements}

Not applicable

\section{References}

1. Nitsche MA, Paulus W. Excitability changes induced in the human motor cortex by weak transcranial direct current stimulation. J Physiol. 2000;527(3):633-9.

2. Nitsche MA, Paulus W. Sustained excitability elevations induced by transcranial DC motor cortex stimulation in humans. Neurology. 2001;57(10):1899. 
3. Machado DG da S, Unal G, Andrade SM, Moreira A, Altimari LR, Brunoni AR, et al. Effect of transcranial direct current stimulation on exercise performance: A systematic review and meta-analysis. Brain Stimulat. 2019;12(3):593-605.

4. Abdelmoula A, Baudry S, Duchateau J. Anodal transcranial direct current stimulation enhances time to task failure of a submaximal contraction of elbow flexors without changing corticospinal excitability. Neuroscience. 2016;322:94-103.

5. Angius L, Pageaux B, Hopker J, Marcora SM, Mauger AR. Transcranial direct current stimulation improves isometric time to exhaustion of the knee extensors. Neuroscience. 2016;339:363-75.

6. Cogiamanian F, Marceglia S, Ardolino G, Barbieri S, Priori A. Improved isometric force endurance after transcranial direct current stimulation over the human motor cortical areas. Eur J Neurosci. 2007;26(1):242-9.

7. Barwood MJ, Butterworth J, Goodall S, House JR, Laws R, Nowicky A, et al. The effects of direct current stimulation on exercise Performance, pacing and perception in temperate and hot environments. Brain Stimulat. 2016;9(6):842-9.

8. Kan B, Dundas JE, Nosaka K. Effect of transcranial direct current stimulation on elbow flexor maximal voluntary isometric strength and endurance. Appl Physiol Nutr Metab. 2013;38(7):734-9.

9. Muthalib M, Kan B, Nosaka K, Perrey S. Effects of transcranial direct current stimulation of the motor cortex on prefrontal cortex activation during a neuromuscular fatigue task: an fNIRS study. In: Van Huffel S, Naulaers G, Caicedo A, Bruley DF, Harrison DK, editors. Oxygen Transport to Tissue XXXV. Springer New York; 2013. p. 73-9.

(Advances in Experimental Medicine and Biology).

10. Angius L, Pascual-Leone A, Santarnecchi E. Chapter 17 - Brain stimulation and physical performance. In: Marcora S, Sarkar M, editors. [Progress in Brain Research]. Elsevier; 2018. p. 317-39.

11. Sidhu SK, Cresswell AG, Carroll TJ. Corticospinal responses to sustained locomotor exercises: moving beyond single-joint studies of central fatigue. Sports Med. 2013;43(6):437-49.

12. McNeil CJ, Giesebrecht S, Gandevia SC, Taylor JL. Behaviour of the motoneurone pool in a fatiguing submaximal contraction. J Physiol. 2011;589(14):3533-44.

13. Taylor JL, Todd G, Gandevia SC. Evidence for a supraspinal contribution to human muscle fatigue. Clin Exp Pharmacol Physiol. 2006;33(4):400-5.

14. Nitsche MA, Seeber A, Frommann K, Klein CC, Rochford C, Nitsche MS, et al. Modulating parameters of excitability during and after transcranial direct current stimulation of the human motor cortex. J Physiol. 2005;568(1):291-303.

15. Thomas R, Stephane P. Prefrontal cortex oxygenation and neuromuscular responses to exhaustive exercise. Eur J Appl Physiol. 2008;102(2):153-63.

16. Pageaux B. The psychobiological model of endurance performance: an effort-based decision-making theory to explain self-paced endurance performance. Sports Med. 
2014;44(9):1319-20.

17. Robertson CV, Marino FE. A role for the prefrontal cortex in exercise tolerance and termination. J Appl Physiol. 2016;120(4):464-6.

18. Bennabi D, Pedron S, Haffen E, Monnin J, Peterschmitt Y, Van Waes V. Transcranial direct current stimulation for memory enhancement: from clinical research to animal models. Front Syst Neurosci. 2014;8.

19. Koechlin E, Ody C, Kouneiher F. The architecture of cognitive control in the human prefrontal cortex. Science. 2003;302(5648):1181-5.

20. MacDonald AW, Cohen JD, Stenger VA, Carter CS. Dissociating the role of the dorsolateral prefrontal and anterior cingulate cortex in cognitive control. Science. 2000;288(5472):1835-8.

21. Rutherford HJV, Lindell AK. Thriving and surviving: approach and avoidance motivation and lateralization. Emot Rev. 2011;3(3):333-43.

22. Lattari E, Campos C, Lamego MK, Passos S de S, Neto GM, Rocha NB, et al. Can transcranial direct current stimulation improve muscle power in individuals with advanced resistance training experience? J Strength Cond Res. 2017.

23. Seidel O, Ragert P. Effects of transcranial direct current stimulation of primary motor cortex on reaction time and tapping performance: a comparison between athletes and nonathletes. Front Hum Neurosci. 2019;13.

24. Grosprêtre S, Gimenez P, Martin A. Neuromuscular and electromechanical properties of ultra-power athletes: the traceurs. Eur J Appl Physiol. 2018;118(7): 1361-71.

25. Grosprêtre S, Martin A. H reflex and spinal excitability: methodological considerations. Journal of Neurophysiology. 2011;107(6):1649-54.

26. Edwards DJ, Cortes M, Wortman-Jutt S, Putrino D, Bikson M, Thickbroom G, et al. Transcranial direct current stimulation and sports performance. Front Hum Neurosci. $2017 ; 11$.

\section{Figures}




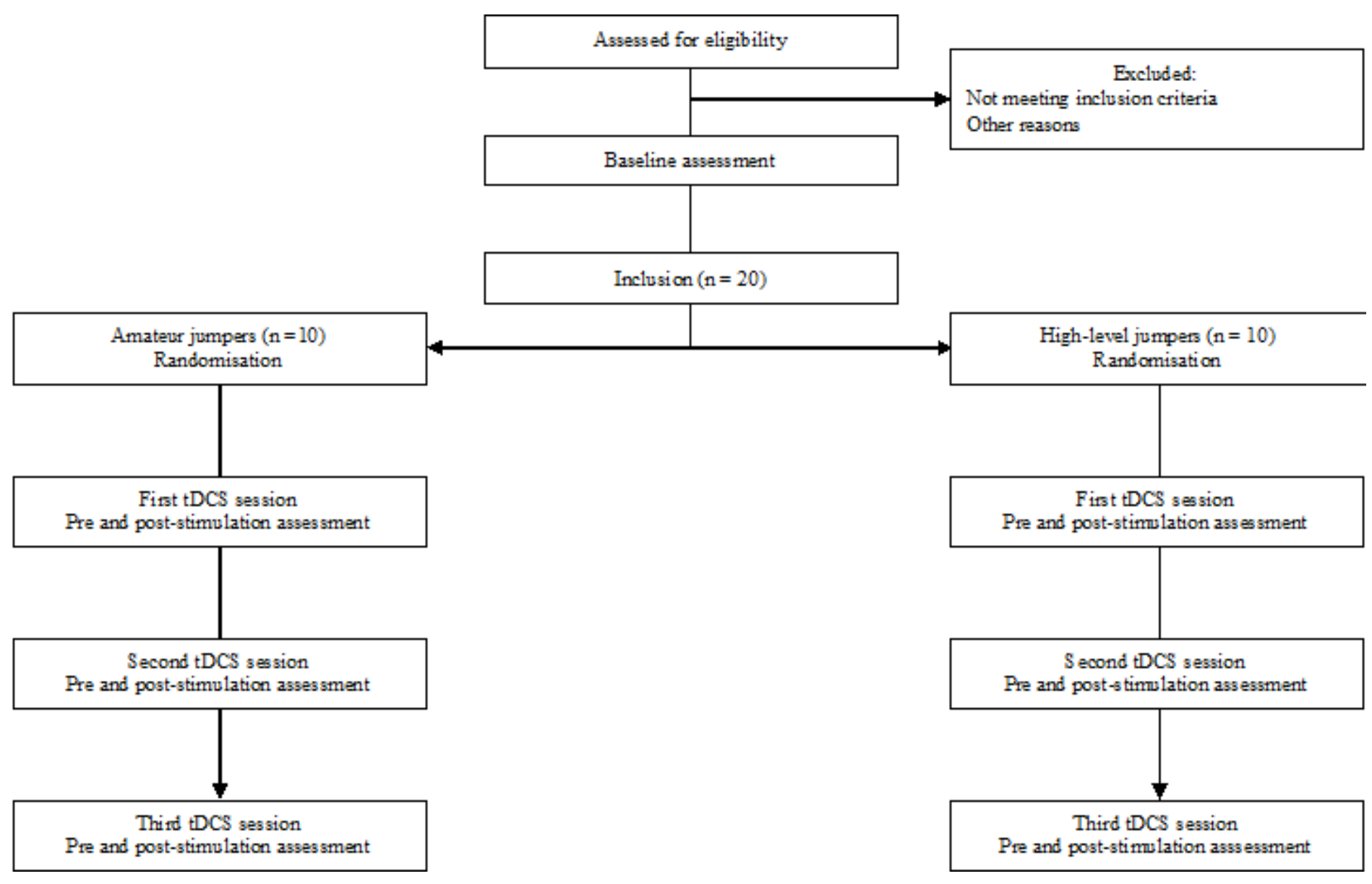

\section{Figure 1}

Study flow diagram (part A) 


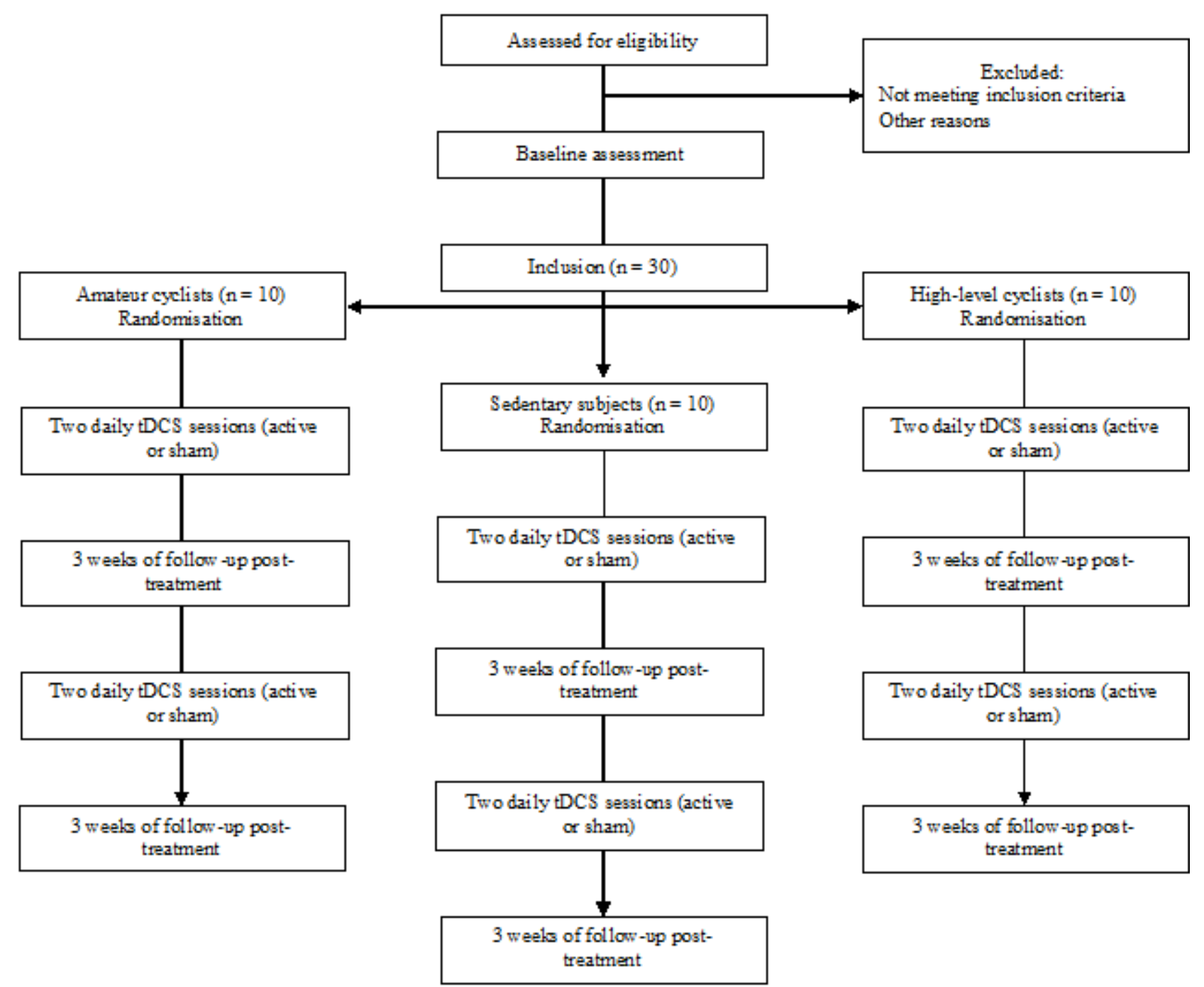

Figure 2

Study flow diagram (Part B) 
Figure 3: Randomised cross-over design for COMPETE (part A) (Standard Protocol Items: Recommendations for Interventional Trials (SPIRIT) Figure).

\begin{tabular}{|c|c|c|c|c|c|}
\hline \multirow[b]{4}{*}{ TIMEPOINT (DAYS) } & \multicolumn{5}{|c|}{ STUDY PROTOCOL } \\
\hline & \multirow{3}{*}{$\begin{array}{c}\text { Phase } 1 \\
\text { Enrolment } \\
\text { Day -1 }\end{array}$} & \multicolumn{2}{|c|}{ Phase 2} & Phase 3 & Phase 4 \\
\hline & & Inclusion & \multicolumn{3}{|c|}{ Follow-up } \\
\hline & & Day 0 & Visit 1 & Visit 2 & Visit 3 \\
\hline ENROLMENT & \multirow{4}{*}{$\mathrm{x}$} & \multirow{4}{*}{$\begin{array}{l}x \\
x\end{array}$} & & & \\
\hline Presentation of study & & & & & \\
\hline Informed consent & & & & & \\
\hline $\begin{array}{l}\text { Randomised } \\
\text { allocation }\end{array}$ & & & & & \\
\hline $\begin{array}{l}\text { INTERVENTIONS } \\
\text { Active } \mathrm{tDCS} \\
\left(2 \mathrm{~mA} / 25 \mathrm{~cm}^{2}, 20 \mathrm{~min},\right. \\
\text { dIPEC) } \\
\text { Active } \mathrm{tDCS} \\
\left(2 \mathrm{~mA} / 25 \mathrm{~cm}^{2}, 20 \mathrm{~min}, \mathrm{Ml}\right) \\
\text { Sham tDCS }\end{array}$ & & & \multicolumn{3}{|c|}{$\begin{array}{l}\text { SEQUENCE DETERMINED BY } \\
\text { RANDOMISATION }\end{array}$} \\
\hline $\begin{array}{l}\text { ASSESSMENTS } \\
\text { Clinical evaluation } \\
\text { Primary outcome. } \\
\text { Height or length of jumps } \\
\text { Secondary outcome: } \\
\text { Newromuscular parameters, BIS- } \\
10, \text { BART, MCQ, EERRT, Go/No- } \\
\text { Go task, Stroop task, OIDS-CI6, } \\
\text { OIDS-SRI6, Poivting task }\end{array}$ & & $\mathrm{x}$ & $\mathrm{x}$ & $x$ & $x$ \\
\hline
\end{tabular}

BART Balloon Analog Riak Task, BIS-10 Barratt Impulsiveness Scale-10, dUPEG Dorsolateral Prefrontal Cortex, EEfRTEffort Expenditure for Rewards Task, MI Primary motor cortex, MCQ Monetary Choice Questionnaire, QIDS-C16 16-Item Quick Inventory of Depressive Symptomatology, Clinician Rating , QIDS-SRI6 16-Item Quick Inventory of Depressive Symptomatology, Self-Report

\section{Figure 3}

Randomised cross-over design for COMPETE (part A) (Standard Protocol Items: Recommendations for Interventional Trials (SPIRIT) Figure). BART Balloon Analog Risk Task, BIS-10 Barratt Impulsiveness Scale-10, dIPFC Dorsolateral Prefrontal Cortex, EEfRT Effort Expenditure for Rewards Task , M1 Primary motor cortex, MCQ Monetary Choice Questionnaire, QIDS-C16 16-Item Quick Inventory of Depressive Symptomatology, Clinician Rating , QIDS-SR16 16-Item Quick Inventory of Depressive Symptomatology, Self-Report 
Figure 4. Randomized cross-over design for COMPETE (part B) (Standard Protocol Items: Recommendations for Interventional Trials

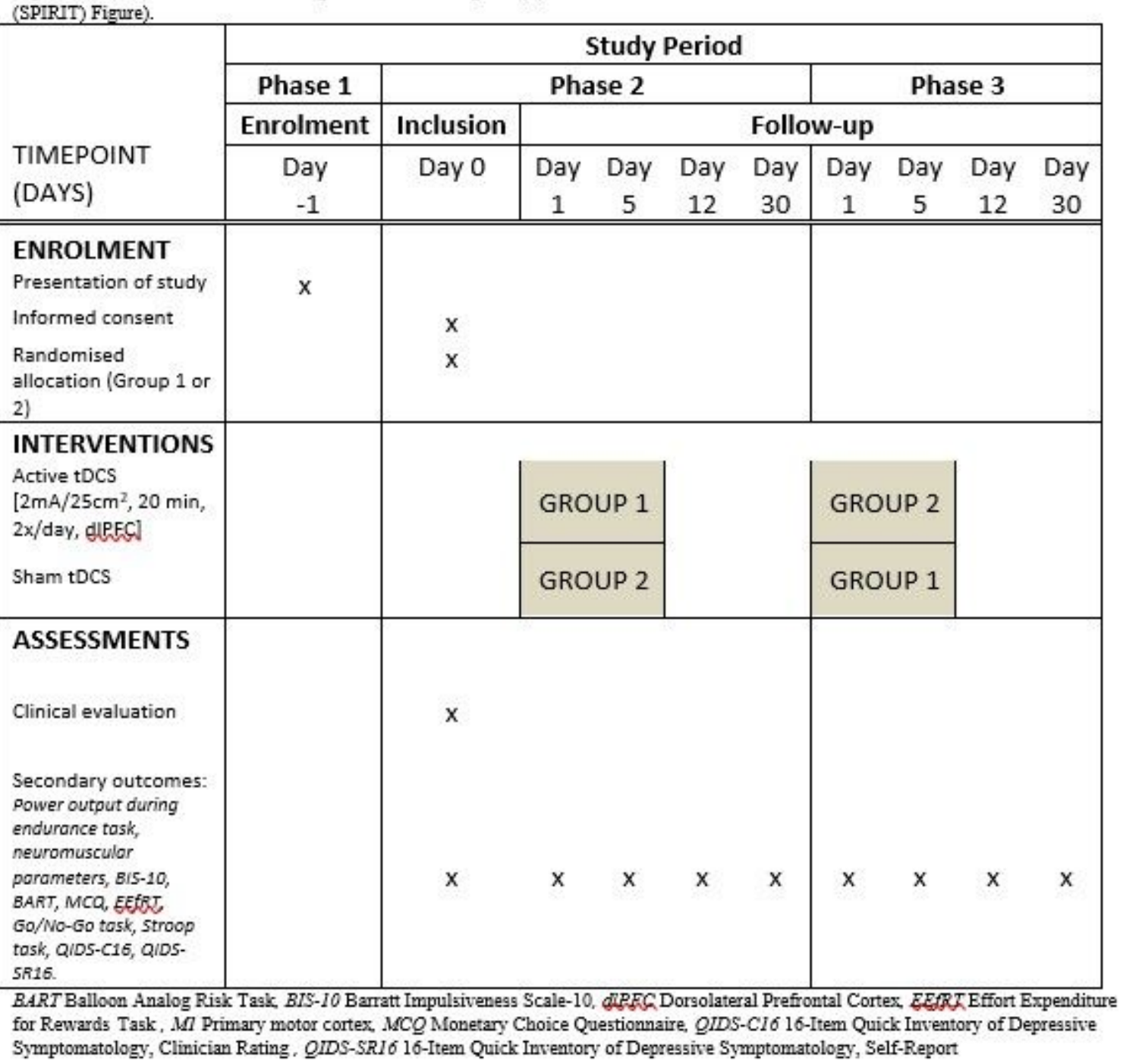

\section{Figure 4}

Randomized cross-over design for COMPETE (part B) (Standard Protocol Items: Recommendations for Interventional Trials (SPIRIT) Figure). BART Balloon Analog Risk Task, BIS-10 Barratt Impulsiveness Scale-10, dIPFC Dorsolateral Prefrontal Cortex, EEfRT Effort Expenditure for Rewards Task , M1 Primary motor cortex, MCQ Monetary Choice Questionnaire, QIDS-C16 16-Item Quick Inventory of Depressive Symptomatology, Clinician Rating , QIDS-SR16 16-Item Quick Inventory of Depressive Symptomatology, Self-Report

\section{Supplementary Files}

This is a list of supplementary files associated with this preprint. Click to download.

- ChecklistSpiritCompete.doc 
- BMCTV5studyprotocolCOMPETE.docx

Page 23/23 